\title{
Necessary and Sufficient Conditions of Fourier Coefficients of a Function and Its Applications
}

\author{
R.Suryanarayana ${ }^{1}$ and Ch. Gopala Rao $^{2}$ \\ Dept. of Mathematics, GMR Institute of Technology, Rajam-532127, AP, India
}

Abstract: In this paper, we present necessary and sufficient condition that a function $f(x)=\sum_{n=1}^{\infty} a_{n} \cos n x$, where the coefficient $a_{n}(n=1,2,3, \ldots \ldots)$ are quasi-monotone to be of class $L(\phi, \psi)$. Finally we discussed the applications of the Fourier series

\section{Introduction}

Fourier series are of great importance in both theoretical and applied mathematics. This paper will focus on the study of order of decrease of Fourier coefficients of a function belonging to different subclasses of class $L_{\phi}(\phi \geq 1)$ represents one of the fundamental issues of Fourier theory. This paper deals with the Fourier coefficients of a function of class $L(\phi, \psi)$, where $1 \leq \phi<\infty,-1<\phi \psi<\phi-1$. Of the many possible methods of estimating complex-valued functions, Fourier series are especially attractive because uniform convergence of the Fourier series (as more terms are added) is guaranteed for continuous, bounded functions.

\section{Basic Results}

We represent the main statements needed for representation of the result of this paper.

Def 2.1. A sequence $\left\{\lambda_{n}\right\}$ is quasi-monotone if $\lambda_{n}>0$ and $n^{-\tau} \lambda_{n} \downarrow 0$ for some $\tau>0$.

Def 2.2. Let $1 \leq \mu<\infty$, we say that the function $\mathrm{f}$ with period $2 \pi$ is in class $L_{\phi}$, if

So

$$
\begin{aligned}
\|f\|_{\phi} & =\left\{\int_{0}^{2 \pi}|f(x)|^{\phi} d x\right\}^{1 / \phi}<\infty \\
L_{\phi} & =\frac{f(x)}{\|f(x)\|_{\phi}}=\left\{\int_{0}^{2 \pi}|f(x)|^{\phi} d x\right\}^{1 / \phi}<\infty
\end{aligned}
$$

Def.2.3. A function $\mathrm{f}(\mathrm{x})$ is said to belong to the class $L(\phi, \psi)$, if $\|f\|_{\phi \psi}=\left\{\int_{0}^{\pi}|f(x)|^{\phi}(\sin x)^{\phi \psi} d x\right\}^{1 / \phi}<\infty$, where $1 \leq \phi<\infty,-1<\phi \psi<\phi-1$.

So $\quad L(\phi, \psi)=\frac{f(x)}{\|f(x)\|_{\phi, \psi}}=\left\{\int_{0}^{\pi}|f(x)|^{\phi}(\sin x)^{\phi \psi} d x\right\}^{1 / \phi}<\infty$.

The following statement gives necessary condition adequate that is necessary to complete Fourier coefficients in order that function belongs to class $L_{\phi}(L(\phi, \psi)$.

Theorem 2.1. Let $1 \leq \phi \leq 2$ and $q=\frac{\phi}{\phi-1}(2 \leq q \leq \infty)$. The following estimate holds true

1. If $f \in L_{\phi}$ and $\left\{c_{n}\right\}^{\infty}{ }_{n=-\infty}$ are Fourier coefficients of function, then

$$
\left\{\sum_{|n|=0}^{\infty}\left|c_{n}\right|^{q}\right\}^{1 / q} \leq A(\phi)\|f\|_{\phi}
$$

2. If $\left\{c_{n}\right\}_{n=-\infty}^{\infty}$ is sequence of numbers such that $\sum_{|n|=0}^{\infty}\left|c_{n}\right|^{\phi}<\infty$

Then there exists a function $f \in L_{q}$ with Fourier coefficients $\left\{c_{n}\right\}$ the inequality

$$
\|f\|_{\phi} \leq A^{\prime}(q)\left\{\sum_{|n|=0}^{\infty}\left|c_{n}\right|^{q}\right\}^{1 / q} \text { holds true. }
$$

Theorem 2.2. The necessary and sufficient condition that $\sum_{n=1}^{\infty} a_{n} \cos n x, a_{n} \downarrow 0$ be the Fourier series of a function $f \in L_{\phi}, \phi>1$ is that the series $\sum_{n=1}^{\infty} a_{n}{ }^{\phi} n^{\phi-2}<\infty$.

Theorem. 2.3. The necessary and sufficient condition that the $\sum_{n=1}^{\infty} a_{n} \cos n x$, where $\left\{a_{n}\right\}$ is positive and quasi monotone be Fourier series of a function $f \in L(\phi, \psi)$, where $1 \leq \phi<\infty,-1<\phi \psi<\phi-1$ is that the series $\sum_{n=1}^{\infty}\left(a_{n}\right)^{\phi} n^{\phi-\phi \psi-2}<\infty$.

In [1] given the following theorem concerning the Fourier coefficients of a function belonging to $L_{\phi}$ class. Theorem 2.4. Let $f \in L_{\phi}, \phi>1$, function given with Fourier series $f(x)=\sum_{n=1}^{\infty} a_{n} \cos n x, \quad a_{n} \downarrow 0$. Then $\frac{S_{1}}{1}, \frac{S_{2}}{2}, \frac{S_{3}}{3}, \ldots \ldots$ are also Fourier coefficients of a function of class $L_{\phi}$, where $S_{n}=\sum_{k=1}^{n} a_{k}$.

Lemma 2.1. If $\left\{a_{n}\right\}$ is positive and quasi - monotone, then $\left\{A_{n}\right\}=\frac{1}{n} \sum_{k=1}^{n} a_{k}$ is also positive and quasi monotone. 


\section{Main Results}

Theorem 3.1. Let $f(x) \in L(\phi, \psi), 1 \leq \phi<\infty,-1<\phi \psi<\phi-1$, function given with Fourier series $f(x)=\sum_{n=1}^{\infty} a_{n} \cos n x$, where $\left\{a_{n}\right\}$ is positive and quasi - monotone.

Then the series $\sum_{n=1}^{\infty} A_{n} \cos n x$, where $\left\{A_{n}\right\}=\frac{1}{n} \sum_{k=1}^{n} a_{k}$ will be Fourier series of a function $\mathrm{F}(\mathrm{x})$ of class $L(\phi, \psi)$.

Proof: Let $f(x)=L(\phi, \psi), 1 \leq \phi<\infty,-1<\phi \psi<\phi-1$, function given with Fourier series $f(x)=$ $\sum_{n=1}^{\infty} a_{n} \cos n x$, where $\left\{a_{n}\right\}$ is positive and quasi monotone.

Since $\left\{a_{n}\right\}$ is positive and quasi monotone and due to Lemma $2.1,\left\{A_{n}\right\}=\frac{1}{n} \sum_{k=1}^{n} a_{k}$ is positive and quasi monotone. To proof of this theorem we have to show that $\sum_{n=1}^{\infty}\left(A_{n}\right)^{\phi} n^{\phi-\phi \psi-2}<\infty$, then by theorem (2.3) follows that the series $\sum_{n=1}^{\infty} A_{n} \cos n x$ is a Fourier series of a function $\mathrm{F}(\mathrm{x})$ of class $L(\phi, \psi)$.

Let

$$
f_{1}(x)=\int_{0}^{x} f(x) d x \text { and } f_{2}(x)=\int_{0}^{x} f_{1}(x) d x
$$

Then,

$$
f_{2}(x)=\sum_{k=1}^{\infty} a_{k}(1-\cos k x) k^{-2} \geq \sum_{k=1}^{n} a_{k}(1-\cos k x) k^{-2}
$$

For $\frac{\pi}{4(n+1)} \leq x \leq \frac{\pi}{4 n}$

We have $f_{2}(x)=\rho_{1} \cdot n^{-2} \cdot \sum_{k=1}^{\infty} a_{k} \geq \rho_{1} \cdot n^{-1} A_{n}$ for same constant $\rho_{1}$.

So that $\quad A_{n} \leq \rho . n . f_{2}(x)$ for same constant $\rho$.

Thus

$$
\begin{aligned}
\sum_{n=1}^{\infty}\left(A_{n}\right)^{\phi} n^{\phi-\phi \psi-2} & \leq \rho \sum_{n=1}^{\infty}\left(f_{2}(x)\right)^{\phi} n^{\phi-\phi \psi-2} \\
& =\rho \sum_{n=1}^{\infty} n^{\phi-\phi \psi-2} \min _{\frac{\pi}{4(n+1)} \leq x \leq \frac{\pi}{4 n}}\left(f_{2}(x)\right)^{\phi} \\
& \leq \rho \sum_{n=1}^{\infty} \int_{\frac{\pi}{4(n+1)}}^{\frac{\pi}{4 n}}(\sin x)^{\phi \psi-\phi}\left[\frac{f_{2}(x)}{x}\right]^{\phi} d x \\
& =\rho \int_{0}^{\frac{\pi}{4 n}}(\sin x)^{\phi \psi-\phi}\left[x^{-1} f_{2}(x)\right]^{\phi} d x \\
& \leq \rho(\psi, \phi) \int_{0}^{\frac{\pi}{4}}(\sin x)^{\phi \psi-\phi}\left[x^{-1} f_{1}(x)\right]^{\phi} d x \\
& \leq \rho(\psi, \phi) \int_{0}^{\frac{\pi}{4}}(\sin x)^{\phi \psi}[f(x)]^{\phi} d x<\infty .
\end{aligned}
$$

A similar method may be used to estimate

$$
\begin{aligned}
& \int_{0}^{\frac{\pi}{4}}(\sin x)^{\phi \psi}[f(x)]^{\phi} d x<\infty . \\
& \sum_{n=1}^{\infty} n^{\phi-\phi \psi-2}\left(A_{n}\right)^{\phi}<\infty .
\end{aligned}
$$

Hence the theorem .

Theorem 3.2. Let $(x)=\sum_{n=1}^{\infty} a_{n} \cos n x$, where $\left\{a_{n}\right\}$ is positive and quasi monotone. Then a necessary and sufficient condition that $\sum_{n=1}^{\infty} a_{n} \cos n x$ be the Fourier series of a function $f(x) \in L(\phi, \psi)$ is that $\sum_{n=1}^{\infty} A_{n} \cos n x$ to be the Fourier series of a function $\mathrm{F}(\mathrm{x})$ be belonging to $L(\phi, \psi)$ where $1 \leq \phi<\infty,-1<$ $\phi \psi<\phi-1$ and $\left\{A_{n}\right\}=\frac{1}{n} \sum_{k=1}^{n} a_{k}$.

Proof: The necessary part follows from Theorem 3.1 as a particular case.

Sufficiency: Suppose that $\sum_{n=1}^{\infty} A_{n} \cos n x$ is a fourier series of a function $f(x) \in L(\phi, \psi)$

Since $\left\{a_{n}\right\}$ is positive and quasi monotone, then by lemma (2.1) follows $\left\{A_{n}\right\}$ is positive and quasi monotone. Hence by theorem (2.3) we have $\sum_{n=1}^{\infty}\left(A_{n}\right)^{\phi} n^{\phi-\phi \psi-2}<\infty$. Since sequence $\left\{a_{n}\right\}$ is positive and quasi monotone for some constant $\tau>0$, sequence $n^{-\tau} a_{n} \downarrow 0$, and for some constant $\rho_{1}>0$ we have $n^{-\tau} a_{n} \leq$ $\rho_{1} k^{-\tau} a_{k}$ for $\mathrm{k}<\mathrm{n}$, then it follows that

$$
\begin{aligned}
A_{n} & =\frac{1}{n} \sum_{k=1}^{n} a_{k}=\frac{1}{n} \sum_{k=1}^{n} k^{-\tau} a_{k} k^{\tau} \geq \frac{1}{\rho_{1}} \frac{1}{n} n^{-\tau} a_{n} \sum_{k=1}^{n} k^{\tau} \\
& =\frac{1}{\rho_{1}} \frac{1}{n} n^{-\tau} a_{n} n n^{\tau}=\frac{1}{\rho_{1}} a_{n} \quad \Rightarrow a_{n} \leq \rho_{1} A_{n}
\end{aligned}
$$

So that $\quad \sum_{n=1}^{\infty}\left(a_{n}\right)^{\phi} n^{\phi-\phi \psi-2} \leq\left(\rho_{1}\right)^{\phi} \sum_{n=1}^{\infty}\left(A_{n}\right)^{\phi} n^{\phi-\phi \psi-2}<\infty$.

Hence by theorem (2.3) $f(x) \in L(\phi, \psi)$ and consequently $\sum_{n=1}^{\infty} a_{n} \cos n x$ is the Fourier series of a function $f(x)$.

\section{Applications Of Fourier Series}

Fourier series simplify the analysis of periodic, real valued functions. Specifically, it can break up a periodic function into an infinite series of sine and cosine waves. This property makes Fourier series very useful in many applications.

Consider the common differential equation given by:

$$
x^{\prime \prime}(t)+a x^{\prime}(t)+b=f(t)
$$


This equation describes the motion of a damped harmonic oscillator that is driven by some function $\mathrm{f}(\mathrm{t})$. It can be used to model an extensive variety of physical phenomena, such as a driven mass on a spring, an analog circuit with a capacitor, resistor, and inductor, or a string vibrated at some frequency. There are two parts to the solution of equation (4.1). The first part is a transient that fades away (generally) fairly quickly. When the transient is gone, what remains is the steady-state solution.

The physical property of oscillating systems that makes Fourier Analysis useful is the property of superposition in other words, suppose the driving force $f_{1}(t)$ along with some initial conditions, produces some steady state solution $x_{1}(t)$, and that another driving force $f_{2}(t)$ produces the steady state solution $x_{2}(t)$. Then the driving force $f_{3}(t)=f_{1}(t)+f_{2}(t)$ produces the steady-state response $x_{2}(t)=x_{1}(t)+x_{2}(t)$.

Then, since we can represent any period driving function as a Fourier series, and it is a simple matter to find the steady-state solution to a sinusoidally driven oscillator, we can find the response to the arbitrary driving function

$$
f(x)=a_{0}+\sum a_{n} \cos n x+\sum b_{n} \sin n x
$$

So suppose we had our square wave equation, where $f(t)$ is the square wave function. We could then decompose the square wave into sinusoidal components as follows:

$$
\begin{aligned}
c_{n}= & \frac{1}{2 \pi} \int_{-\pi}^{\pi} s(x) e^{-i n x}=\frac{1}{2 \pi} \int_{0}^{\pi} e^{-i n x} \\
= & \frac{i}{2 n \pi}\left(e^{i n \pi}-1\right) \\
c_{-n} & =\frac{-i}{2 n \pi}\left(e^{-i n \pi}-1\right)
\end{aligned}
$$

and then just combine the $c_{n}$ and $c_{-n}$ terms as before. The result would be an infinite sum of sin and cosin terms of the form in equation (2). The steady-state response of the system to the square wave would then just be the sums of the steady-state responses to the sinusoidal components of the square wave.

The basic equations of the Fourier series led to the development of the Fourier transform, which can decompose a non-periodic function much like the Fourier series decomposes a periodic function. Because this type of analysis is very computation-intensive, different Fast-Fourier Transform algorithms have been devised, which lower the order of growth of the number of operations from order $\left(\mathrm{N}^{2}\right)$ to order $(\mathrm{n} \log (\mathrm{n}))$.

With these new techniques, Fourier series and Transforms have become an integral part of the toolboxes of mathematicians and scientists. Today, it is used for applications as diverse as file compression, signal processing in communications and astronomy, acoustics, optics, and cryptography.

\section{REFERENCES}

[1] G.H. Hardy, Not on some points in integral calculus, Messenger of Mathematics, 58, (1929), 50-52.

[2] N.K Bari, Trigonometriçeskie rjade, Moskva, 1961.

[3] R. Askey and R. Wainger, Integrability theorems for Fourier series, Duke Mathematical Journal, 33(1), (1966), 223-228.

[4] A.K. Gaur, A theorem for Fourier coefficients of a function of class LP, International Journal of Mathematics and Mathematical Sciences, 13(4), (1990), 721-726.

[5] Eric W. Weisstein. "Fourier Series." From Math world-a Wolfram Web Resource. http://mathworld.wolfram.com/FourierSeries.html. 